\title{
Isolated extramedullary cutaneous relapse despite concomitant severe graft-vs.-host disease and tissue chimerism analysis in a patient with acute lymphoblastic leukemia after allogeneic hematopoietic stem cell transplantation: A case report
}

\author{
BULENT KANTARCIOGLU ${ }^{1}$, HUSEYIN SAFFET BEKOZ ${ }^{1}$, YELIZ DUVARCI OGRET ${ }^{2}$, \\ ASLI CAKIR ${ }^{3}$, DEMET KIVANC ${ }^{1}$, FATMA SAVRAN OGUZ ${ }^{2}$ and DENIZ SARGIN ${ }^{1}$ \\ ${ }^{1}$ Department of Internal Medicine, Division of Hematology, Istanbul Medipol University; ${ }^{2}$ Istanbul Medical Faculty, \\ Department of Medical Biology, Istanbul University; ${ }^{3}$ Department of Pathology, \\ Istanbul Medipol University, Istanbul 34214, Turkey
}

Received June 16, 2016; Accepted September 23, 2016

DOI: $10.3892 /$ mco.2016.1052

\begin{abstract}
Allogeneic hematopoietic stem cell transplantation (allo-HSCT) is a potentially curative treatment option for patients with acute lymphoblastic leukemia (ALL). The curative potential of allo-HSCT for ALL is, in part, due to the graft-vs.-leukemia (GVL) effect, in addition to the intensive conditioning chemo-radiotherapy. However, relapse remains the major cause of treatment failure following allo-HSCT for ALL. In the allo-HSCT setting, testing for genetic markers of hematopoietic chimerism has become a part of the routine diagnostic program. Routine chimerism analysis is usually performed in peripheral blood or bone marrow; in fact, little is known about the value of tissue chimerism in patients with extramedullary relapse (EMR) after the allo-HSCT setting. The present study reports on, a case of a patient with ALL who experienced isolated cutaneous EMR despite ongoing graft-vs.-host disease (GVHD), and the results of peripheral
\end{abstract}

Correspondence to: Dr Bulent Kantarcioglu, Department of Internal Medicine, Division of Hematology, Istanbul Medipol University, 1 Bagcilar, Istanbul 34214, Turkey

E-mail: bulentkantarcioglu@gmail.com

Abbreviations: ALL, acute lymphoblastic leukemia; AML, acute myeloid leukemia; allo-HSCT, allogeneic hematopoietic stem cell transplantation; CR1, first complete remission; DLI, donor lymphocyte infusion; ECPP, extracorporeal photopheresis; EMR, extramedullary relapse; GMALL, German multicenter acute lymphoblastic leukemia; GVHD, graft-vs.-host disease; GVL, graft-vs.-leukemia; MMF, mycophenolate mofetil; MUD, matched unrelated donor; MAC, myeloablative conditioning; OS, overall survival; PCR, polymerase chain reaction; STR, short tandem repeats

Key words: acute lymphoblastic leukemia, allogeneic stem cell transplantation, extramedullary relapse, tissue chimerism blood and skin tissue chimerism studies using multiplex polymerase chain reaction (PCR) of short tandem repeats (STR-PCR). The present case demonstrates that, although complete remission and/or chimerism may be achieved in the bone marrow, chimerism achieved at the tissue level, and the subsequent GVL effect, may be limited, despite concomitant severe GVHD following allo-HSCT. Our tissue chimerism analysis results provide a good example of how skin tissue may be a 'sanctuary' site for effector cells of GVL, despite active GVHD and complete hematopoetic chimerism.

\section{Introduction}

Allogeneic hematopoietic stem cell transplantation (allo-HSCT) is a potentially curative treatment option for patients with acute lymphoblastic leukemia (ALL). The curative potential of allo-HSCT for ALL is, in part, due to the graft-vs.-leukemia (GVL) effect, in addition to the intensive conditioning chemo-radiotherapy. However, relapse remains the major cause of treatment failure following allo-HSCT for ALL (1). ALL relapse usually occurs in the bone marrow, although a significant rate of extramedullary relapse (EMR) following allo-HSCT has been reported either alone or concomitantly with bone marrow relapse. In general, the incidence of EMR is higher in patients with ALL compared with those with acute myeloid leukemia (AML). Ge et al (2) reported that the incidence of EMR was $12.9 \%$ in patients with ALL, in which isolated EMR occurred in $7.9 \%$ of them. However, the mechanism of EMR is very poorly understood; and the prognosis of these patients is generally poor (2).

In the allo-HSCT setting, testing for genetic markers of hematopoietic chimerism has become a part of the routine diagnostic program. Chimerism testing permits early prediction and documentation of successful engraftment, and facilitates early detection of impending graft rejection. For patients who undergo transplantation for the treatment of malignant hematological disorders, monitoring of chimerism can provide an early indication of incipient disease relapse, and enable an 
assessment of the ability to demonstrate the GVL effect to be made (3). Routine chimerism analysis is usually performed in peripheral blood or bone marrow; in fact, little is known about the value of tissue chimerism in patients with EMR following the allo-HSCT setting.

In the present study, a case of a patient with ALL who experienced isolated cutaneous EMR despite ongoing graft-vs.-host disease (GVHD), and the results of peripheral blood and skin tissue chimerism studies using multiplex polymerase chain reaction (PCR) of short tandem repeats (STR-PCR). This case demonstrates that, although complete remission and/or chimerism may be achieved in the bone marrow, chimerism achieved at the tissue level, and the subsequent GVL effect, may be limited, despite concomitant severe GVHD following allo-HSCT.

\section{Case report}

A 52 year-old female presented to a Turkish hospital with erythematous skin nodules on her trunk, arms and face with bilateral pleural effusion and hepatosplenomegaly in October 2012. The skin and bone marrow biopsy were consistent with precursor T-cell acute lymphoblastic leukemia. The patient was started on induction chemotherapy using the German multicenter acute lymphoblastic leukemia (GMALL) 05/93 protocol (4), and remission was achieved. The treatment was subsequently continued with early consolidation, reinduction and late consolidation treatments. However, skin lesions recurred in December 2013. The patient's bone marrow examination was clean at that time. Due to progressive disease reinduction, chemotherapy with the identical protocol and skin-directed psoralen and ultraviolet A radiation (PUVA) treatment was started in January 2014. However, progression in the skin lesions occurred with this treatment, and a relapse was also evident in the bone marrow. In March 2014, treatment with a FLAG-ida chemotherapy regimen was started. The disease was also resistant to this chemotherapy, since remission was achieved in bone marrow, but the nodular skin lesions remained. The patient was then treated with two cycles of clofarabine in combination with high-dose cytosine arabinoside in May 2014. However, the disease was also refractory to this treatment, since, although the bone marrow was kept in remission, the nodular skin lesions returned at the time of hematological recovery. A matched related donor for allogeneic bone marrow transplantation was not identified for this patient, and so she was referred to our clinic at the Istanbul Medipol University for a matched unrelated donor (MUD) allo-HSCT in June 2014.

The patient underwent unmanipulated peripheral blood SCT from a 10/10 MUD on July 1, 2014. The myeloablative conditioning (MAC) regimen was with total body irradiation (TBI; 12 Gy in six fractions) and cyclophosphamide (120 mg/kg). Cyclosporin A, short-course methotrexate and standard dose anti-thymocyte globulin (ATG) were used for acute GVHD prophylaxis. Allo-HSCT was performed without any difficulties, and neutrophil and thrombocyte engraftment was achieved on day +16 of the transplantation. However, a diffuse erythematous rash appeared on the patient's trunk and bilateral extremities at the time of engraftment. The results of the liver function tests were also abnormal. Acute
GVHD of the skin and liver was suspected, and a treatment with prednisolone started at a dose of $2 \mathrm{mg} / \mathrm{kg} / \mathrm{day}$. The skin lesions disappeared, and results of the liver function tests were normalized with this treatment. At day +63 of the transplantation, acute GVHD progressed, with a tapering of steroid doses. In view of this development, three doses of pulse steroid treatment (10 mg/kg/day) and mycophenolate mofetil (MMF) were added to the treatment. In the follow-up, acute GVHD had progressed again, despite pulse steroid and MMF treatment, and so $\beta$-human chorionic gonadotropin ( $\beta$-HCG; pregnyl) was used at a dose of $187.5 \mathrm{mg} /$ day for 2 weeks. However, on day +118 , the skin lesions of GVHD had progressed, and the levels of the liver enzymes were again increased. Furthermore, diarrhea had been added to the patient's symptoms. The results of the previous skin and liver biopsies were consistent with GVHD. Consequently, extracorporeal photopheresis (ECPP) in combination with mesencyhmal stem cell infusion was performed for the patient. With this treatment, the GVHD was able to be controlled successfully. At day +143 of the transplantation, new nodular lesions were appearing on the chest and torso of the patient (Fig. 1). The biopsy of these lesions was consistent with T-cell ALL infiltration. Notably, GVHD was still ongoing, and the bone marrow was still in remission at that time. Isolated EMR was considered, presenting with skin involvement. Nelarabine treatment was planned for the patient. However, the condition of the patient deteriorated with the infection, and she succumbed to hospital-acquired bacteriemia with sepsis at +200 days of allo-HSCT.

During the patient's follow-up, no sign of relapse in the bone marrow biopsy examinations of the patient was observed. The peripheral blood STR-PCR chimerism results were all consistent with complete chimerism at days $+30,+90$ and +180 , which supported the bone marrow remission (Table I). STR-PCR chimerism analysis in paraffin-embedded skin biopsies at days $+16,+63,+143$ and +198 were also retrospectively performed. The skin biopsies that were taken at days +16 , +63 and +198 were from the areas of skin GVHD, whereas the biopsy at day +143 was taken directly from the nodular skin lesion of leukemia relapse. It is noteworthy that the chimerism status was not chimeric (recipient type) at days +16 and +63 , but it was mixed chimeric at days +143 and +198 (Table II).

\section{Discussion}

The case presented in the present study details a heavily pretreated patient with high-risk features, who underwent an allo-HSCT and suffered from a very aggressive course of disease that progressed rapidly, to be controlled by a very active immune response.

The vast majority (up to 90\%) of adult patients with ALL are able to achieve remission with the use of intensive induction chemotherapy. However, relapse eventually occurs, and the 5 year overall survival (OS) is $42-63 \%$ for adolescents and young adults, $24.1 \%$ for patients between the ages of $40-59$, and $17.7 \%$ for patients between the ages of 60-69 (5). Historically, age, a high leukocyte count at presentation, poor response to treatment, T-cell immunophenotype, cytogenetic profile of the disease and extranodal presentation of the disease have been considered adverse clinical prognostic factors in adult ALL. In relapsed patients, long-term disease-free survival and cure 
Table I. Results of peripheral blood chimerism studies using PCR-STR analysis.

\begin{tabular}{|c|c|c|c|c|c|}
\hline STR locus & Donor & $\begin{array}{l}\text { Recipient } \\
\text { (pre-Tx) }\end{array}$ & $\begin{array}{c}\text { Recipient } \\
(\text { post-Tx }+30)\end{array}$ & $\begin{array}{c}\text { Recipient } \\
(\text { post-Tx }+90)\end{array}$ & $\begin{array}{c}\text { Recipient } \\
(\text { post-Tx }+180)\end{array}$ \\
\hline D21S11 & $28,32.2$ & 29,31 & $28,32.2$ & $28,32.2$ & $28,32.2$ \\
\hline D7S820 & 10,10 & 9,10 & 10,10 & 10,10 & 10,10 \\
\hline CSF1PO & 9,10 & 11,11 & 9,10 & 9,10 & 9,10 \\
\hline D3S1358 & 15,18 & 16,18 & 15,18 & 15,18 & 15,18 \\
\hline TH01 & 6,9 & 7,10 & 6,9 & 6,9 & 6,9 \\
\hline D13S317 & 12,14 & 11,12 & 12,14 & 12,14 & 12,14 \\
\hline D16S539 & 9,11 & 8,13 & 9,11 & 9,11 & 9,11 \\
\hline D2S1338 & 24,24 & 20,20 & 24,24 & 24,24 & 24,24 \\
\hline D19S433 & $13.2,14$ & 13,15 & $13.2,14$ & $13.2,14$ & $13.2,14$ \\
\hline VWA & 14,17 & 17,18 & 14,17 & 14,17 & 14,17 \\
\hline TPOX & 11,12 & 8,10 & 11,12 & 11,12 & 11,12 \\
\hline D1851 & 12,14 & 16,18 & 12,14 & 12,14 & 12,14 \\
\hline D5S818 & 11,11 & 11,13 & 11,11 & 11,11 & 11,11 \\
\hline \multirow[t]{2}{*}{ FGA } & 19,21 & 24,25 & 19,21 & 19,21 & 19,21 \\
\hline & PCR-STK & & Complete chimer & Complete chimeri & Complete chimeric \\
\hline
\end{tabular}

Pre-Tx, pre-transplant; post-Tx, post-transplant; PCR-STR, polymerase chain reaction-short tandem repeats.

Table II. Result of skin tissue chimerism studies by PCR-STR analysis.

\begin{tabular}{|c|c|c|c|c|c|c|}
\hline STR locus & Donor & $\begin{array}{l}\text { Recipient } \\
\text { (pre-Tx) }\end{array}$ & $\begin{array}{c}\text { Recipient } \\
(\text { post-Tx }+16)\end{array}$ & $\begin{array}{c}\text { Recipient } \\
(\text { post-Tx }+63)\end{array}$ & $\begin{array}{c}\text { Recipient } \\
(\text { post-Tx }+143)\end{array}$ & $\begin{array}{c}\text { Recipient } \\
(\text { post-Tx }+198)\end{array}$ \\
\hline D3S1358 & 15,18 & 16,18 & 16,18 & 16,18 & $16,18,15,18$ & $16,18,15,18$ \\
\hline TH01 & 6,9 & 7,7 & 7,7 & 7,7 & $7,7,6,9$ & $7,7,6,9$ \\
\hline D21S11 & 28,29 & 29,31 & 29,31 & 29,31 & $29,31,28,29$ & $29,31,28,29$ \\
\hline D18S51 & 22,22 & 25,26 & 25,26 & 25,26 & $25,26,22,22$ & $25,26,22,22$ \\
\hline PENTA-E & 15,17 & 7,15 & 7,15 & 7,15 & $7,15,15,17$ & $7,15,15,17$ \\
\hline D5S818 & 11,11 & 11,12 & 11,12 & 11,12 & $11,12,11,11$ & $11,13,11,11$ \\
\hline D13S317 & 11,11 & 11,12 & 11,12 & 11,12 & $11,12,11,11$ & $11,12,11,11$ \\
\hline D7S820 & 10,10 & 9,10 & 9,10 & 9,10 & $9,10,10,10$ & $9,10,10,10$ \\
\hline D16S539 & 9,11 & 8,13 & 8,13 & 8,13 & $8,13,9,11$ & $8,13,9,11$ \\
\hline CSF1PO & 6,13 & 7,7 & 7,7 & 7,7 & $7,7,6,13$ & $7,7,6,13$ \\
\hline PENTA-D & 10,10 & 10,13 & 10,13 & 10,13 & $10,13,10,10$ & $10,13,10,10$ \\
\hline VWA & 14,14 & 16,18 & 16,18 & 16,18 & $16,18,14,14$ & $16,18,14,14$ \\
\hline TPOX & 11,11 & 8,10 & 8,10 & 8,10 & $8,10,11,11$ & $8,10,11,11$ \\
\hline \multirow[t]{2}{*}{ FGA } & 21,21 & 24,25 & 24,25 & 24,25 & $24,25,21,21$ & $24,25,21,21$ \\
\hline & \multicolumn{2}{|c|}{ Result of PCR-STR analysis } & Not chimeric & Not chimeric & Mixed chimeric & Mixed chimeric \\
\hline
\end{tabular}

Pre-Tx, pre-transplant; post-Tx, post-transplant; PCR-STR, polymerase chain reaction-short tandem repeats.

may be obtained in 7-24\% of patients with only allo-HSCT (6). Cutaneous involvement with ALL occurs rarely, with an incidence between $0.5-3 \%$ (7).

For this reason, allo-HSCT, despite its limitations and toxicities, is an accepted therapy for adults. In addition to historical prognostic factors, certain factors, including the performance status of the patient, availability and type of a transplant donor, persistence of residual disease at transplantation, the cytomegalovirus seropositivity of the recipient, the type of conditioning regimen, using a T-cell-depleted graft, and the presence and grade of GVHD during transplantation, were defined as the predictors of allo-HSCT outcome (8-12). In our patient, the age at presentation, T-cell phenotype of the disease, extranodal involvement, refractoriness to conventional chemotherapy and absence of a donor in first complete remission (CR1) were the most important predictors of relapse. In contrast, the performance status was good in our patient, therefore it was possible to perform a MAC allo-HSCT without a T-cell-depleted graft. 


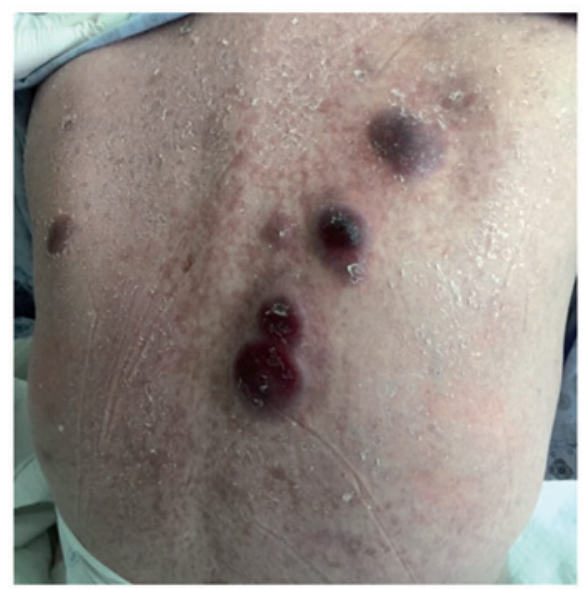

Figure 1. Cutaneous leukemic nodules on the torso of the patient.

However, Duval et al (13) published an analysis of their patients who underwent transplantation during relapse or primary induction failure that were reported to the Center for International Blood and Marrow Transplant Research (CIBMTR) between 1995 and 2004. From a total of 2,255 patients, 582 were diagnosed with ALL. The 3-year OS rate was $16 \%$ (13). The present study demonstrated that HSCT is able to induce long-term survival in patients with ALL, even if they are not in CR. In parallel with this outcome, Kozlowski et al (14) reported an analysis of 76 adults with relapsed ALL within the Swedish Adult Acute Leukemia Registry. In that study, being $>35$ years of age at diagnosis, and relapse within 18 months, were negative prognostic factors. The OS rates at 3 and 5 years were 22 and 15\%, respectively. The authors mentioned a high cure rate via intensive reinduction chemotherapy and allo-HSCT in a population of relapsed ALL patients (14). Although these results are not very satisfactory, they do confirm that allo-HSCT is able to overcome the adverse prognostic impact of relapsed/refractory ALL. Accordingly, we were successful in achieving a complete remission with complete hematopoetic chimerism in our patient through performing an allo-HSCT.

On the other hand, the GVL effect is one of the most important factors of anti-tumor activity, after allo-HSCT. GVL effects are frequently associated with GVHD in ALL patients $(15,16)$. In this regard, Passweg et al (17) reported a comparison of the effects of GVHD on relapse rates in B-lineage and T-lineage ALL. Their study confirmed the anti-leukemic effect of GVHD in ALL, and this effect was similar in T- and B-lineage ALL. In our patient, there was an apparent resistance to graft-vs.-ALL activity, as evidenced by rapid disease progression despite severe and resistant GVHD. The GVL effect maintained marrow remission without being capable of preventing EMR. In this regard, our tissue chimerism analysis results confirmed the absence of active GVL in the cutaneous tissue of our patient.

It is noteworthy that developments in medicine are changing the natural course of ALL. In this regard, although the overall frequency of relapse is lower following allo-HSCT, an increased trend in EMR has been reported in $\operatorname{ALL}(18,19)$. These observations suggested that the GVL effect at extramedullary sites may be less prominent compared with that in the bone marrow. However, other factors, including the nature of the leukemic blasts, status of acute leukemia at hematopoietic cell transplantation, and the conditioning regimen, may also influence the frequency of EMR. In the literature, central nervous system (CNS) relapse is the most common subtype of EMR after allo-HSCT. Patients with high-risk cytogenetics, an advanced disease status, a history of EM leukemia prior to allo-HSCT, hyperleukocytosis at diagnosis, receipt of peripheral blood stem cells (PBSCs) and the male gender are reported risk factors for EMR following allo-HSCT (2). Accordingly, the cutaneous leukemic involvement of our patient was problematical from the beginning of the disease. Relapse occurred despite intensive chemotherapy and ongoing GVHD. Unfortunately, the prognosis for EMR following allo-HSCT is poor, and efficient treatment strategies are lacking in this setting. Radiotherapy, salvage chemotherapy, second transplantation and donor lymphocyte infusion (DLI) have all been utilized, but the choice of the therapeutic strategy that is optimal remains controversial. DLI was not a treatment option for our patient due to the active GVHD.

Among recipients of allo-HSCT, donor stem cell engraftment in non-hematopoietic tissues has been observed by several groups. Graft-derived cells with an epithelial phenotype have been described in skin, the gastrointestinal tract, and in liver of human allo-HSCT recipients (20). However, the exact origin of these cells and their pattern of engraftment in response to injury have yet to be elucidated. In this regard, Willemze et al (21) investigated the occurrence of endothelial and epithelial cell chimerism skin biopsies of allo-HSCT recipients using the fluorescence in situ hybridization (FISH) method. Endothelial cell chimerism was found in $25 \%$ of the biopsies, and increased in time, particularly in patients with acute GVHD. Epithelial cell chimerism was found in $85 \%$ of the biopsies, and was not correlated with the time interval following SCT or with tissue damage caused by GVHD (21). These results contrasted with observations made by Murata et al (22), who observed endothelial cell chimerism (using FISH method) shortly after the start of acute GVHD. However, in the study by Imanishi et al (23) published during the same year, donor-derived DNA in the fingernails of allo-HSCT recipients was identified only in 9 of 21 cases using the STR-PCR method. The time from transplantation to sampling was in excess of 300 days in that study (23). In support of this study, Pearce et al (24) also reported donor chimerism in four of eight cases in the fingernails of reduced intensity conditioning regimen (RIC) allo-HSCT patients using the STR-PCR method. It should be emphasized that the latter two studies investigated the contribution of donor-derived cells in fingernails, i.e. a tissue without blood cells. In our patient, STR-PCR chimerism analysis was performed in paraffin-embedded skin biopsies that contained blood cells. The chimerism status was not chimeric (recipient type) at days +16 or +63 following the transplantation, until cutaneous EMR occurred on day +143 . This finding emphasized that complete chimerism was achieved in the hematopoietic tissue: The skin of our patient was a 'sanctuary' site for GVL effector cells up to day +63 . This result supports a previously published report by our group (25). In the present study, the mixed chimerism obtained at day +143 may be associated with either an immune booster reaction secondary to the leukemia relapse, or it may 
be associated with the natural course of chimerism achieved at the tissue level. In neither situation was it sufficient to control the disease in our patient.

In conclusion, the present case study has presented a patient with ALL with isolated cutaneous EMR despite active severe GVHD. Our tissue chimerism analysis results have illustrated a good example that skin tissue may be a 'sanctuary' site for effector cells of GVL, despite active GVHD and complete hematopoetic chimerism.

\section{References}

1. Marks DI, Alonso L and Radia R: Allogeneic hematopoietic cell transplantation in adult patients with acute lymphoblastic leukemia. Hematol Oncol Clin North Am 28: 995-1009, 2014.

2. Ge L, Ye F, Mao X, Chen J, Sun A, Zhu X, Qiu H, Jin Z, Miao M, $\mathrm{Fu} \mathrm{C}$, et al: Extramedullary relapse of acute leukemia after allogeneic hematopoietic stem cell transplantation: Different characteristics between acute myelogenous leukemia and acute lymphoblastic leukemia. Biol Blood Marrow Transplant 20 1040-1047, 2014.

3. Martinelli G, Trabetti E, Farabegoli P, Testoni N, Bandini G, Motta MR, Vittone A, Terragna C, Pignatti PF and Tura S: Early detection of bone marrow engraftment by amplification of hypervariable DNA regions. Haematologica 82: 156-160, 1997.

4. Gökbuget N, Hoelzer D, Arnold R, Böhme A, Bartram CR, Freund M, Ganser A, Kneba M, Langer W, Lipp T, et al: Treatment of adult ALL according to protocols of the German multicenter study group for adult ALL (GMALL). Hematol Oncol Clin North Am 14: 1307-1325, ix, 2000.

5. Pulte D, Jansen L, Gondos A, Katalinic A, Barnes B, Ressing M, Holleczek B, Eberle A and Brenner H; GEKID Cancer Survival Working Group: Survival of adults with acute lymphoblastic leukemia in Germany and the United States. PLoS One 9: e85554, 2014.

6. Frey NV and Luger SM: How I treat adults with relapsed or refractory Philadelphia chromosome-negative acute lymphoblastic leukemia. Blood 126: 589-596, 2015.

7. Nabhan C, Tolentino A, Meyer A and Tallman MS: Cutaneous involvement with B-lineage acute lymphoblastic leukemia. Leuk Lymphoma 53: 987-989, 2012.

8. Doney K, Hägglund H,Leisenring W, Chauncey T, Appelbaum FR and Storb R: Predictive factors for outcome of allogeneic hematopoietic cell transplantation for adult acute lymphoblastic leukemia. Biol Blood Marrow Transplant 9: 472-481, 2003.

9. Hunault M, Harousseau JL, Delain M, Truchan-Graczyk M, Cahn JY, Witz F, Lamy T, Pignon B, Jouet JP, Garidi R, et al: Better outcome of adult acute lymphoblastic leukemia after early genoidentical allogeneic bone marrow transplantation (BMT) than after late high-dose therapy and autologous BMT: A GOELAMS trial. Blood 104: 3028-3037, 2004.

10. Yanada M, Matsuo K, Suzuki T and Naoe T: Allogeneic hematopoietic stem cell transplantation as part of postremission therapy improves survival for adult patients with high-risk acute lymphoblastic leukemia: A metaanalysis. Cancer 106: 2657-2663, 2006.

11. Marks DI, Pérez WS, He W, Zhang MJ, Bishop MR, Bolwell BJ, Bredeson CN, Copelan EA, Gale RP, Gupta V, et al: Unrelated donor transplants in adults with Philadelphia-negative acute lymphoblastic leukemia in first complete remission. Blood 112 426-434, 2008

12. Fielding AK, Rowe JM, Richards SM, Buck G, Moorman AV, Durrant IJ, Marks DI, McMillan AK, Litzow MR, Lazarus HM, et al: Prospective outcome data on 267 unselected adult patients with Philadelphia chromosome-positive acute lymphoblastic leukemia confirms superiority of allogeneic transplantation over chemotherapy in the pre-imatinib era: Results from the International ALL Trial MRC UKALLXII/ECOG2993. Blood 113: 4489-4496, 2009.
13. Duval M, Klein JP, He W, Cahn JY, Cairo M, Camitta BM, Kamble R, Copelan E, de Lima M, Gupta V, et al: Hematopoietic stem-cell transplantation for acute leukemia in relapse or primary induction failure. J Clin Oncol 28: 3730-3738, 2010.

14. Kozlowski P, Åström M, Ahlberg L, Bernell P, Hulegårdh E, Hägglund $\mathrm{H}$, Karlsson $\mathrm{K}$, Markuszewska-Kuczymska A, Tomaszewska-Toporska B, Smedmyr B and Hallböök H: High curability via intensive reinduction chemotherapy and stem cell transplantation in young adults with relapsed acute lymphoblastic leukemia in Sweden 2003-2007. Haematologica 97: 1414-1421, 2012.

15. Sullivan KM, Weiden PL, Storb R, Witherspoon RP, Fefer A, Fisher L, Buckner CD, Anasetti C, Appelbaum FR, Badger C, et al: Influence of acute and chronic graft-versus-host disease on relapse and survival after bone marrow transplantation from HLA-identical siblings as treatment of acute and chronic leukemia. Blood 73: 1720-1728, 1989.

16. Horowitz MM, Gale RP, Sondel PM, Goldman JM, Kersey J, Kolb HJ, Rimm AA, Ringdén O, Rozman C, Speck B, et al: Graft-versus-leukemia reactions after bone marrow transplantation. Blood 75: 555-562, 1990.

17. Passweg JR, Tiberghien P, Cahn JY, Vowels MR, Camitta BM, Gale RP, Herzig RH, Hoelzer D, Horowitz MM, Ifrah N, et al: Graft-versus-leukemia effects in T lineage and B lineage acute lymphoblastic leukemia. Bone Marrow Transplant 21: 153-158, 1998.

18. Lee JH, Choi SJ, Lee JH, Seol M, Lee YS, Ryu SG, Park CJ, Chi HS, Lee MS, Yun S, et al: Anti-leukemic effect of graft-versus-host disease on bone marrow and extramedullary relapses in acute leukemia. Haematologica 90: 1380-1388, 2005.

19. Shi JM, Meng XJ, Luo Y, Tan YM, Zhu XL, Zheng GF, He JS, Zheng WY, Xie WZ, Li L, et al: Clinical characteristics and outcome of isolated extramedullary relapse in acute leukemia after allogeneic stem cell transplantation: A single-center analysis. Leuk Res 37: 372-377, 2013.

20. Hong YC, Liu HM, Chen PS, Chen YJ, Lyou JY, Hu HY, Yi MF, Lin JS and Tzeng CH: Hair follicle: A reliable source of recipient origin after allogeneic hematopoietic stem cell transplantation. Bone Marrow Transplant 40: 871-874, 2007.

21. Willemze AJ, Bakker AC, von dem Borne PA, Bajema IM and Vossen JM: The effect of graft-versus-host disease on skin endothelial and epithelial cell chimerism in stem-cell transplant recipients. Transplantation 87: 1096-1101, 2009.

22. Murata H, Janin A, Leboeuf C, Soulier J, Gluckman E, Meignin V and Socie G: Donor-derived cells and human graft-versus-host disease of the skin. Blood 109: 2663-2651, 2007.

23. Imanishi D, Miyazaki Y, Yamasaki R, Sawayama Y, Taguchi J, Tsushima H, Fukushima T, Yoshida S, Sasaki H, Hata T and Tomonaga M: Donor-derived DNA in fingernails among recipients of allogeneic hematopoietic stem-cell transplants. Blood 110: 2231-2234, 2007.

24. Pearce L, Lim ZY, Usai M, Ho AY, Mufti GJ and Pagliuca A: Mixed donor chimaerism in recipient fingernails following reduced-intensity conditioning haematopoietic SCT. Bone Marrow Transplant 42: 361-362, 2008.

25. Kalayoglu-Besisik S, Oguz FS, Aydin F, Duvarci Y, Sargin D and Carin M: Tissue chimerism analysis after donor lymphocyte infusion: A case of deceptive evidence. Hematology 13: 92-94, 2008. 